\title{
Trimaximal Mixing with One Texture Zero from Type II Seesaw and $\Delta(54)$ Family Symmetry
}

\author{
M. A. Loualidi \\ LPHE-Modeling and Simulations, Faculty of Science, Mohammed V University in Rabat, 10090, Morocco
}

\begin{abstract}
We propose a neutrino model based on a $\Delta(54)$ flavor symmetry suitable for explaining the current neutrino oscillation data. Neutrino masses arise from the type II seesaw mechanism where the $\Delta(54)$ field assignments has led to a simple neutrino mass matrix with one texture zero and which satisfies the magic symmetry consistent with the well-known trimaximal mixing matrix. We found interesting predictions concerning neutrino masses and mixing. In particular, only the normal neutrino mass hierarchy and the lower octant of the atmospheric angles are allowed in this model. The model predicts as well that the $C P$ conserving values for the Dirac $C P$ phase $\delta_{C P}$ are not allowed and thus, $C P$ is always violated in the neutrino sector. We have also investigated the sum of absolute neutrino masses from cosmological observations, the effective Majorana mass $m_{\beta \beta}$ from neutrinoless double beta decay experiments, and the electron neutrino mass $m_{\beta}$ from beta decays where we found that the obtained range of $m_{\beta \beta}$ can be tested by several experiments in the near future.
\end{abstract}

Keywords: neutrino model building, trimaximal mixing, one texture zero, flavor symmetries

DOI: 10.31526/ACP.BSM-2021.25

\section{INTRODUCTION}

Despite the major progress in neutrino physics over the last two decades, there are many questions and properties related to neutrinos that are yet to be addressed such as: (i) whether neutrinos are their own antiparticles or not, which is equivalent to asking whether these particles are Majorana or Dirac fermions; (ii) the presence of $C P$ violation in the chargeless sector; (iii) the absolute scale of neutrino masses; (iv) the neutrino mass ordering problem; and (v) the octant of the atmospheric mixing angle. The investigation of these features provides us with a clear evidence that we need to look beyond the standard model (SM) of electroweak interactions.

There are dozens of experiments looking at neutrinos from different angles [1, 2], each using different insights to help unraveling the properties of this elusive particle. Theoretically, one of the best ways to generate neutrino masses is via the so-called seesaw mechanism where the smallness of neutrino mass is explained by the presence of super-heavy partners. There are three popular realizations of this mechanism: type I seesaw with right-handed neutrinos [3], type II seesaw with heavy $S U(2)_{L}$ scalar triplets [4] and type III see-saw with $S U(2)_{L}$ fermion triplets [5] ${ }^{1}$. On the other hand, one of the most popular patterns of the leptonic mixing is acquired by the trimaximal $\left(\mathrm{TM}_{2}\right)$ mixing matrix which predicts non-zero reactor angle $\theta_{13}$ and non-maximal atmospheric angle $\theta_{23}$. This mixing matrix is defined as [9]

$$
U_{T M_{2}}=\left(\begin{array}{ccc}
\sqrt{\frac{2}{3}} \cos \theta & \frac{1}{\sqrt{3}} & \sqrt{\frac{2}{3}} \sin \theta e^{-i \sigma} \\
-\frac{\cos \theta}{\sqrt{6}}-\frac{\sin \theta}{\sqrt{2}} e^{i \sigma} & \frac{1}{\sqrt{3}} & \frac{\cos \theta}{\sqrt{2}}-\frac{\sin \theta}{\sqrt{6}} e^{-i \sigma} \\
-\frac{\cos \theta}{\sqrt{6}}+\frac{\sin \theta}{\sqrt{2}} e^{i \sigma} & \frac{1}{\sqrt{3}} & -\frac{\cos \theta}{\sqrt{2}}-\frac{\sin \theta}{\sqrt{6}} e^{-i \sigma}
\end{array}\right) \cdot U_{P}
$$

where $\theta$ parameterizes the deviation from the well known tribimaximal (TBM) ansatz [10], $\sigma$ is an arbitrary phase and $U_{P}=$ $\operatorname{diag}\left(1, e^{i \alpha_{21} / 2}, e^{i \alpha_{31} / 2}\right)$ is a diagonal matrix that encodes the Majorana phases $\alpha_{21}$ and $\alpha_{31}$. In this regard, it is commonly known that the observed pattern of neutrino masses and mixing suggest the existence of symmetries acting in the space of fermion generations. In particular, the TBM mixing can be derived using non-Abelian discrete symmetries and subsequently, $\mathrm{TM}_{2}$ is obtained by rotating the TBM matrix in the 1-3 plane. Moreover, one of the simplest ways to reduce the number of free parameters is by developing neutrino mass matrices with one texture zero $[11,12,13]$. Realizing these types of matrices leads to special correlations among the physical quantities such as mass squared differences, mixing angles and $C P$ phases and hence allows for highly predictive models; see Ref. [14] for models studying neutrino mass matrices with one texture zero and $\mathrm{TM}_{2}$.

Here we present a model where the neutrinos acquire their masses through the type II seesaw mechanism whereas the neutrino flavor structure is provided by the $\Delta(54)$ flavor group. In this setup, three gauge singlet scalar fields (flavons) are required by $\Delta(54)$

\footnotetext{
${ }^{1}$ One must note that small neutrino masses can also be generated through the so-called scotogenic mechanism [6] whereby the neutrino mass problem is connected to dark matter. On the other hand, the phenomenology of scotogenic models is interesting and the new extra degrees of freedom can be tested at present and future TeV-scale colliders [7]. Besides, the problem of neutrino mixing can be addressed as usual by the introduction of discrete symmetry groups, see for instance Ref. [8] and the references therein.
} 
invariance and phenomenological reasons. Furthermore, the leptonic mixing arises from the neutrino sector and thus, the $\Delta(54)$ charge assignments of lepton and scalar fields are chosen in a way that the resulting charged lepton Yukawa matrix is diagonal. Afterwards, the $\Delta(54)$ flavor symmetric construction with a specific choice of flavon alignment leads to highly predictive results and give rise to a neutrino mass matrix with two important features: ( $a$ ) one texture zero reducing the number of free parameters to only three; and $(b)$ satisfies the magic symmetry and thus diagonalized by $\mathrm{TM}_{2}$ mixing matrix in (1.1), see e.g., Ref. [15]. In particular, we concluded that only the normal neutrino mass hierarchy is permitted in our model, while we showed through scatter plots that the atmospheric angle lies in the lower octant. Moreover, it is found that the $C P$ conserving values for the Dirac $C P$ phase $\delta_{C P}$ are not allowed in our model and thus, $C P$ is always violated in the neutrino sector. On the other hand, the phenomenological implications of the light neutrino masses are explored using recent global fit of neutrino oscillation parameters from Ref. [16]. In particular, we have investigated the sum of the three active neutrino masses $\sum m_{i}$ from cosmological observations, the effective Majorana mass $\left|m_{\beta \beta}\right|$ from neutrinoless double beta decay $(0 v \beta \beta)$ experiments, and the electron neutrino mass $m_{\beta}$ from beta decays. It is found that the obtained ranges of $\sum m_{i}$ and $\left|m_{\beta \beta}\right|$ can be tested by different planned future experiments while the predicted range of $m_{\beta}$ requires more enhanced sensitivity of beta decay experiments. This contribution is structured as follows. In section 2 , we give some properties of the $\Delta(54)$ group, then we present our $\Delta(54)$ field assignments. In section 3, we examine in details the neutrino oscillation parameters in our model. In section 3 , we study the phenomenological consequences of the neutrino masses from non-oscillatory experiments. In section 4 , we give our conclusion.

\section{IMPLEMENTATION OF $\Delta(54)$ FLAVOR SYMMETRY}

In this section, we describe the particle content and the introduction of $\Delta(54)$ flavor symmetry in the SM. Before giving the full field spectrum of our model, let us first mention some properties of the $\Delta(54)$ group. First, notice that the discrete flavor $\Delta(54)$ symmetry has ten irreducible representations $\mathbf{R}_{i}$ where in addition to two singlets $\mathbf{1}_{+}$and $\mathbf{1}_{-}$, and four doublets $\mathbf{2}_{1,2,3,4}$, it has four three-dimensional irreducible representations denoted as $\mathbf{3}_{k}$ and their conjugates $\overline{\mathbf{3}}_{k}$ with index $k=1,2$. The tensor products between the representations that are relevant to the present work are as follows

$$
\begin{aligned}
\mathbf{1}_{+} \otimes \mathbf{R}_{i}=\mathbf{R}_{i}, \quad \mathbf{1}_{-} \otimes \mathbf{1}_{-}=\mathbf{1}_{+}, \quad \mathbf{1}_{-} \otimes \mathbf{3}_{1}=\mathbf{3}_{2}, \quad \mathbf{1}_{-} \otimes \overline{\mathbf{3}}_{1}=\overline{\mathbf{3}}_{2} \\
\mathbf{1}_{-} \otimes \mathbf{3}_{2}=\mathbf{3}_{1}, \mathbf{1}_{-} \otimes \overline{\mathbf{3}}_{2}=\overline{\mathbf{3}}_{1}, \quad \mathbf{3}_{2} \otimes \overline{\mathbf{3}}_{2}=\mathbf{1}_{+} \oplus \mathbf{2}_{1} \oplus \mathbf{2}_{2} \oplus \mathbf{2}_{3} \oplus \mathbf{2}_{4} \\
\mathbf{3}_{1} \otimes \overline{\mathbf{3}}_{1}=\mathbf{1}_{+} \oplus \mathbf{2}_{1} \oplus \mathbf{2}_{2} \oplus \mathbf{2}_{3} \oplus \mathbf{2}_{4}, \quad \overline{\mathbf{3}}_{2} \otimes \mathbf{3}_{1}=\mathbf{1}_{-} \oplus \mathbf{2}_{1} \oplus \mathbf{2}_{2} \oplus \mathbf{2}_{3} \oplus \mathbf{2}_{4} \\
\overline{\mathbf{3}}_{1} \otimes \mathbf{3}_{2}=\mathbf{1}_{-} \oplus \mathbf{2}_{1} \oplus \mathbf{2}_{2} \oplus \mathbf{2}_{3} \oplus \mathbf{2}_{4}, \quad \mathbf{3}_{2} \otimes \mathbf{3}_{2}=\overline{\mathbf{3}}_{2} \oplus \overline{\mathbf{3}}_{2} \oplus \overline{\mathbf{3}}_{1} \\
\mathbf{3}_{1} \otimes \mathbf{3}_{1}=\overline{\mathbf{3}}_{2} \oplus \overline{\mathbf{3}}_{2} \oplus \overline{\mathbf{3}}_{1}
\end{aligned}
$$

A complete list of tensor products of $\Delta(54)$ irreducible representations can be found in [17]. Moreover, this non-Abelian group is isomorphic to the semidirect product $S_{3} \times \mathbb{Z}_{3} \times \mathbb{Z}_{3}$, where $S_{3}$ is the symmetric group of degree three. Therefore, $\Delta(54)$ is generated by four elements; two generators $\mathcal{S}$ and $\mathcal{T}$ for the $S_{3}$ group and the remaining two, denoted as $\mathcal{U}$ and $\mathcal{V}$, generate $\mathbb{Z}_{3} \times \mathbb{Z}_{3}$. These four generators satisfy the following identity

$$
\mathcal{S}^{3}=\mathcal{T}^{2}=(\mathcal{S} \mathcal{T})^{2}=\mathcal{U}^{3}=\mathcal{V}^{3}=\mathbf{I}_{\mathbf{i d}}
$$

This group can be utilized as a flavor symmetry, but is rarely invoked in particle physics model building; see reference [18] for a simple extension of the SM using $\Delta(54)$ providing a good description of the neutrino sector.

Now, we present our field content where in our extension with a $\Delta(54)$ symmetry, it can be arranged into two sets: (i) the usual lepton and Higgs fields of the SM but carrying as well charges under $\Delta(54)$; these are the three lepton doublets $L_{i}$ with $L_{1}=$ $\left(v_{e}, e^{-}\right)_{L}, L_{2}=\left(v_{\mu}, \mu^{-}\right)_{L}$ and $L_{3}=\left(v_{\tau}, \tau^{-}\right)_{L}$ which are assigned to the irreducible triplet $3_{2}$ of $\Delta(54)$, the three right-handed leptons $E_{i}=\left(e_{R}, \mu_{R}, \tau_{R}\right)$ assigned to the $\Delta(54)$ triplet $\overline{\mathbf{3}}_{2}$, and finally the Higgs doublet $H=\left(H^{+}, H^{0}\right)$ which transforms as a trivial singlet under the $\Delta(54)$ group. (ii) An extra scalar sector that contains an $S U(2)_{L}$ scalar triplet and flavon fields transforming as gauge singlet which are required by $\Delta(54)$ flavor invariance and phenomenological purposes. The $S U(2)_{L}$ triplet $T$ is responsible for the generation of neutrino masses via type II seesaw mechanism while we have added in total three flavons denoted as $\chi, \phi$ and $\rho$; the first two are needed to produce a mass matrix compatible with the the familiar TBM matrix while the third flavon $\rho$ is needed to produce a neutrino mass matrix with magic symmetry; that is a matrix which is compatible with neutrino mixing matrix of trimaximal form. The $\Delta(54)$ quantum numbers that are relevant for this work are summarized in table (1). As mentioned in the

\begin{tabular}{c|r|r||r|r|r|r|r}
\hline \hline Fields & $L_{i}=\left(L_{1}, L_{2}, L_{3}\right)$ & $E_{i}=\left(e_{R}, \mu_{R}, \tau_{R}\right)$ & $H$ & $T$ & $\chi$ & $\phi$ & $\rho$ \\
\hline$\Delta(54)$ & $\mathbf{3}_{2}$ & $\overline{\mathbf{3}}_{2}$ & $\mathbf{1}_{+}$ & $\mathbf{1}_{-}$ & $\mathbf{3}_{2}$ & $\mathbf{3}_{1}$ & $\mathbf{1}_{-}$ \\
\hline \hline
\end{tabular}

TABLE 1: Quantum numbers of lepton and scalar fields under $\Delta(54)$.

introduction, we will mainly concentrate on the neutrino sector; thus, we perform the present study in the basis where the charged lepton mass matrix is diagonal. In this scenario, the leptonic mixing matrix is the one that diagonalizes the light neutrino mass matrix. Indeed, with the field assignments given in table (1) the Lagrangian of the charged leptons given by

$$
\mathcal{L}_{Y}^{l}=\sum_{l=e, \mu, \tau} Y_{l}^{i j} \bar{L}_{i} E_{j}^{c} H+\text { h.c. }
$$


leads, after performing the relevant $\Delta(54)$ tensor product ${ }^{2} \overline{\mathbf{3}}_{(0,-1,0)} \otimes \mathbf{3}_{(0,-1,0)} \otimes \mathbf{1}_{(1,1,1)}$ and electroweak symmetry breaking, to a diagonal mass matrix $\mathcal{M}^{l}=\operatorname{diag}\left(m_{e}, m_{\mu}, m_{\tau}\right)=\frac{v_{H}}{\sqrt{2}} \operatorname{diag}\left(Y_{e}, Y_{\mu}, Y_{\tau}\right)$ with $v_{H}$ being the vacuum expectation value (VEV) of the Higgs doublet $H$. The hierarchy among these masses can be obtained by invoking the well-known Froggatt-Nielsen mechanism, see Ref. [19] for more details.

\section{NEUTRINO MASSES AND MIXING}

In this section, we build a predictive neutrino model with one texture zero mass matrix based on the $\Delta(54)$ non-Abelian flavor symmetry. The light neutrino masses are generated via the type II seesaw mechanism which requires extending the scalar sector of the SM by a scalar triplet $\vec{T}$ with hypercharge $Y \vec{T}=2 \vec{T}$, and which may be represented by the following traceless complex $2 \times 2$ matrix

$$
T=\vec{\sigma} \vec{T}=\left(\begin{array}{cc}
\frac{T^{+}}{\sqrt{2}} & T^{++} \\
T^{0} & -\frac{T^{+}}{\sqrt{2}}
\end{array}\right)
$$

where $\sigma^{a}$ are $2 \times 2$ Pauli matrices. Moreover, we add three flavon fields- $\chi, \phi$ and $\rho$-carrying quantum numbers under the $\Delta(54)$ symmetry. Now, by using the particle assignments shown in table 1 , the $\Delta(54)$-invariant Lagrangian for neutrinos is given by

$$
-\mathcal{L}_{v}^{I I}=\frac{Y_{\chi}^{i j}}{\Lambda}\left(\bar{L}_{i}^{c} i \sigma_{2} T L_{j}\right) \chi+\frac{Y_{\phi}^{i j}}{\Lambda}\left(\bar{L}_{i}^{c} i \sigma_{2} T L\right)_{i} \phi+\frac{Y_{\phi \rho}^{i j}}{\Lambda^{2}}\left(\bar{L}_{i}^{c} i \sigma_{2} T L_{j}\right) \phi \rho+\text { h.c. }
$$

After electroweak symmetry breaking and flavor symmetry breaking, the triplet $T$ acquires a VEV $v_{T}$ by its neutral component $T^{0}$ while the flavon fields develop VEVs along the directions

$$
\langle\chi\rangle=\left(\begin{array}{c}
v_{\chi} \\
0 \\
0
\end{array}\right),\langle\phi\rangle=\left(\begin{array}{c}
0 \\
v_{\phi} \\
0
\end{array}\right),\langle\rho\rangle=v_{\rho}
$$

The resulting mass matrix for light neutrino masses is expressed as a function of three free parameters

$$
M_{v}^{I I}=\left(\begin{array}{ccc}
a+b & 0 & \varepsilon \\
0 & \varepsilon+b & a \\
\varepsilon & a & b
\end{array}\right) \text { with } a=Y_{\chi} \frac{v_{T} v_{\chi}}{\Lambda}, b=Y_{\phi} \frac{v_{T} v_{\phi}}{\Lambda}, \varepsilon=Y_{\phi \rho} \frac{v_{T} v_{\rho} v_{\phi}}{\Lambda^{2}}
$$

This matrix has the magic symmetry as the sum of the elements in any of its columns or rows is exactly the same. Thus, $M_{v}^{I I}$ is diagonalized by the well-known trimaximal mixing matrix $\mathrm{TM}_{2}$ described by two free parameters-an arbitrary angle $\theta$ and a phase $\sigma$ that will be related later on to the Dirac CP phase-that can be determined using the neutrino oscillation data. To extract the mass eigenvalues, we assume that the parameters $a$ and $b$ are real while $\varepsilon$ is complex; $\varepsilon=|\varepsilon| e^{i \phi_{\varepsilon}}$. This assumption is reasonable since in the limit where $\varepsilon \rightarrow 0$ the neutrino mass matrix — depending on $a, b$-has the TBM form which is CP conserving. Therefore, the diagonalization of $M_{v}^{I I}$ using the trimaximal mixing matrix defined in eq. (1.1)— $U_{T M}^{T} M_{v}^{I I} U_{T M}=\operatorname{diag}\left(\left|m_{1}\right|,\left|m_{2}\right|,\left|m_{3}\right|\right)-l$ leads to the following eigenvalues up to order ${ }^{3} \mathcal{O}\left(\varepsilon^{2}\right)$

$$
\begin{gathered}
\left|m_{1}\right|=\sqrt{(a+b)^{2}-|\varepsilon|\left(\cos \phi_{\varepsilon}\right)(a-b)},\left|m_{2}\right|=\sqrt{(a+b)^{2}+2|\varepsilon| \cos \phi_{\varepsilon}(a+b)} \\
\left|m_{3}\right|=\sqrt{(a-b)^{2}-|\varepsilon|\left(\cos \phi_{\varepsilon}\right)(a-b)}
\end{gathered}
$$

where the following conditions for the diagonalization must be fulfilled

$$
\tan 2 \theta=\sqrt{3} \frac{|\varepsilon| \sqrt{a^{2} \sin ^{2} \phi_{\varepsilon}+b^{2} \cos ^{2} \phi_{\varepsilon}}}{|\varepsilon| b \cos \phi_{\varepsilon}-2 a b} \quad, \quad \tan \sigma=\frac{a}{b} \tan \phi_{\varepsilon}
$$

From these masses, we deduce the expressions for the solar and atmospheric mass-squared differences

$$
\Delta m_{21}^{2}=|\varepsilon|(3 a+b) \cos \phi_{\varepsilon} \quad, \quad \Delta m_{31}^{2}=-4 a b
$$

As for the mixing angles $\theta_{13}, \theta_{23}$ and $\theta_{12}$, they may be expressed in the case of trimaximal mixing as a function of $\theta$ and $\sigma$, we have

$$
\sin ^{2} \theta_{13}=\frac{2}{3} \sin ^{2} \theta \quad, \quad \sin ^{2} \theta_{12}=\frac{1}{3-2 \sin ^{2} \theta} \quad, \quad \sin ^{2} \theta_{23}=\frac{1}{2}-\frac{\sqrt{3} \sin 2 \theta}{2\left(3-\sin ^{2} \theta\right)} \cos \sigma
$$

\footnotetext{
${ }^{2}$ This tensor product contains the $\Delta(54)$ trivial singlet $\mathbf{1}_{+}$as can be checked from eq. (2.2).

${ }^{3}$ See Refs. [20, 21] for more details on the diagonalization of the neutrino mass matrix up to order $\mathcal{O}\left(\varepsilon^{2}\right)$.
} 

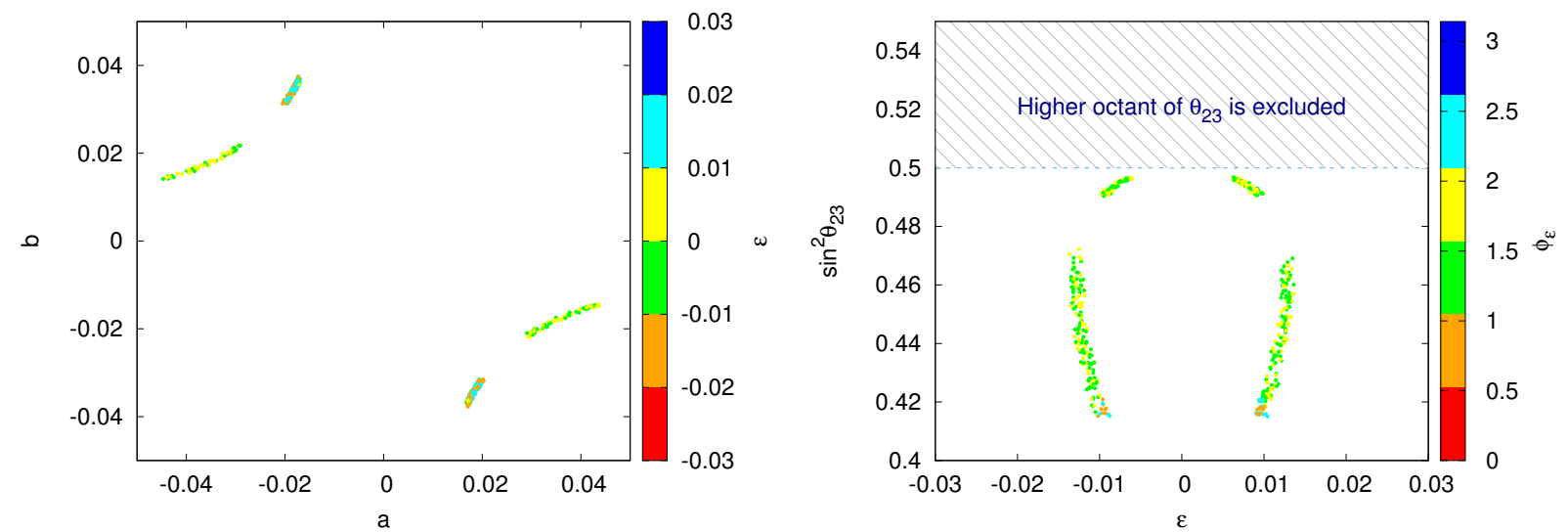

FIGURE 1: Left: The correlation among the parameters $a, b$ and $\varepsilon$. Right: Scatter plot on the plan $\operatorname{of} \sin ^{2} \theta_{23}$ and $\varepsilon$ with the palette showing $\phi_{\varepsilon}$.
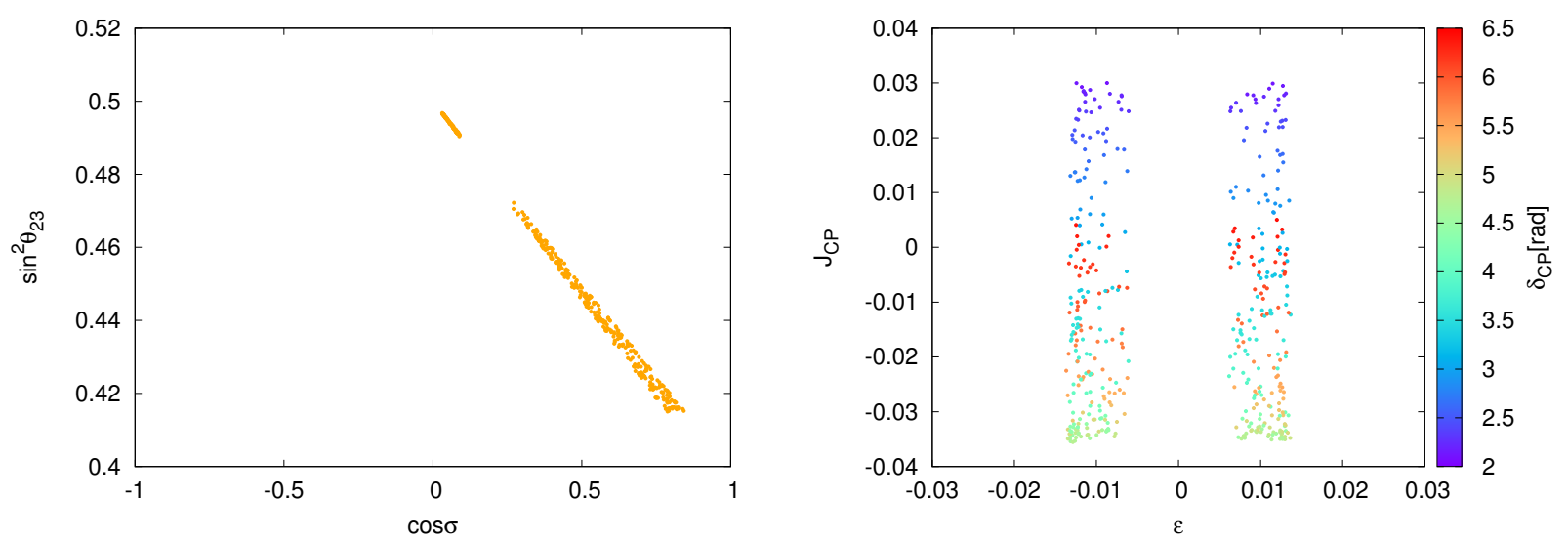

FIGURE 2: Left: The variation of $\sin \theta_{23}^{2}$ as a function of $\cos \sigma$. Right: Scatter plot on the plan of $J_{C P}$ and $\varepsilon$ with the palette showing the Dirac $C P$ phase $\delta_{C P}$.

From the $3 \sigma$ allowed range of $\sin ^{2} \theta_{13}[16]$ and the first equation in (3.12), we find that the range of $\theta$ is given by $0.176 \lesssim \theta[\mathrm{rad}] \lesssim$ 0.193. In the left panel of Fig. 1, we show the correlation among the parameters $a, b$ and $\varepsilon$ where the displayed points satisfy the the $3 \sigma$ experimental values of the oscillation parameters. It is clear from this figure that $a$ and $b$ have opposite signs which means that-according to eq. (3.11) $-\Delta m_{31}^{2}>0$ and thus, the current model predicts the normal hierarchy for neutrino masses which will be focused on in our numerical study. In the right panel of Fig. 1, we display the atmospheric angle as a function of $\varepsilon$ with the palette showing $\phi_{\varepsilon}$. The obtained ranges of these parameters are

$$
\begin{aligned}
0.41503 & \lesssim \sin ^{2} \theta_{23} \lesssim 0.49676, \quad 0.95779 \lesssim \phi_{\varepsilon}[\mathrm{rad}] \lesssim 2.18245 \\
\varepsilon & \in[-0.01363 \rightarrow-0.00597] \cup[0.00597 \rightarrow 0.01363]
\end{aligned}
$$

It is clear from this figure that the atmospheric angle lies in the lower octant $\left(\theta_{23}<\pi / 4\right)$ which is one more important prediction of the model. Another way of getting this prediction is by using the equation of the atmospheric angle in (3.12). In this regard, by using the interval of $\theta$ as an input and we allow the arbitrary phase $\sigma$ to vary randomly in the interval $[-\pi, \pi]$ along with the expressions of the mixing angles in eq. (3.12) and the $3 \sigma$ allowed ranges of neutrino oscillation parameters from Ref. [16], we show in the left panel of Fig. 2 the correlation between $\sin ^{2} \theta_{23}$ and $\cos \sigma$ from which we extract the constrained range of $\sigma$ given as

$$
\sigma \in[-1.5406 \rightarrow 1.5415]
$$

It is clear from this plot that $\cos \sigma>0$ and thus, by plugging the values of $\theta$ and $\cos \sigma$ back in the expression of the atmospheric angle, we get the desired result; $\sin ^{2} \theta_{23}<1 / 2$. On the other hand, a relationship between the arbitrary phase $\sigma$ and the Dirac phase $\delta_{C P}$ can be obtained by means of the Jarlskog invariant parameter defined as $\left.\left.\left.\left.J_{C P}=\operatorname{Im}\left(U_{(} e 1\right) U_{(} \mu 1\right)^{(*)} U_{(} \mu 2\right) U_{(} e 2\right)^{(*)}\right)$. In the PDG parametrization [22], $J_{C P}$ is given in terms of the mixing angles and the Dirac $C P$ phase as

$$
J_{C P}=\frac{1}{8} \sin 2 \theta_{12} \sin 2 \theta_{13} \sin 2 \theta_{23} \cos \theta_{13} \sin \delta_{C P},
$$

while in the case of trimaximal mixing matrix (1.1), $J_{C P}$ takes the following form

$$
\left.J_{C P}\right|_{T M_{2}}=(1 / 6 \sqrt{3}) \sin 2 \theta \sin \sigma
$$



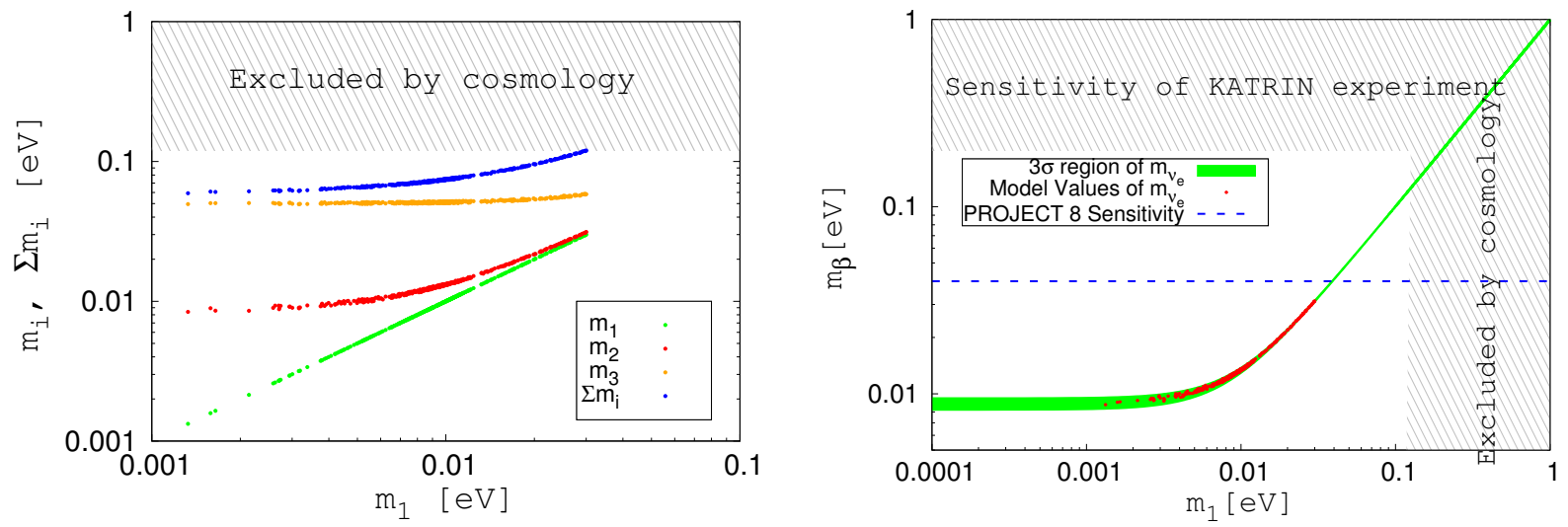

FIGURE 3: Left: Predictions for the absolute neutrino masses $m_{i=1,2,3}$ and their sum $\sum m_{i}$ as a function of the lightest neutrino mass $m_{1}$. The horizontal filled area represent the upper limit on $\sum m_{i}$ from Planck collaboration. Right: $m_{\beta}$ as a function of $m_{1}$. The vertical (horizontal) filled area is disfavored by Planck+BAO (KATRIN) data.

In the right panel of Fig. 2, we plot the Jarlskog invariant parameter as a function of $\varepsilon$ with the color code indicating the range of the Dirac $C P$ phase $\delta_{C P}$ where we find that $J_{C P}$ is predicted to be scattered in the region $-0.0357 \lesssim J_{C P} \lesssim 0.0302$. Furthermore, by using eq. (3.12) and identifying the Jarlskog parameter from the PDG parametrization with the one from trimaximal mixing, we obtain a relation between $\sigma$ and $\delta_{C P}$

$$
\sin \delta_{C P}=\sin \sigma / \sin 2 \theta_{23}
$$

From this equation, we deduce that $\sigma$ and $\delta_{C P}$ are always different from the $C P$ conserving values $n \pi$ where $n$ can be any integer and therefore, it is easy to check from eqs. (3.15) and (3.16) that the Jarlskog invariant parameter does not vanish and consequently, $C P$ violation always occurs in the present model.

\section{PHENOMENOLOGICAL CONSEQUENCES}

Besides neutrino oscillation experiments, information on the absolute neutrino mass scale can be obtained using three different sources:

- (1) Constraints from cosmological observations providing an upper bound on the sum of the three active neutrino masses; $\sum m_{i}=m_{1}+m_{2}+m_{3}$. The present upper bound on $\sum m_{i}$ from the Planck collaboration is given by $\sum m_{i}<0.12 \mathrm{eV}$ at $95 \%$ C.L [23].

- (2) Direct determination of the neutrino mass by measuring the energy spectrum of electrons produced in the $\beta$-decay of nuclei which allows to get information on the effective electron antineutrino mass defined by

$$
m_{\beta}=\left(\sum_{i=1,2,3} m_{i}^{2}\left|U_{e i}\right|^{2}\right)^{1 / 2} .
$$

The current limit from tritium beta decay is given by the KATRIN project, which aims at a detection of $m_{\beta}$ with a sensitivity of $0.2 \mathrm{eV}$ [24].

- (3) Search for $0 v \beta \beta$ decay processes having a decay amplitude proportional to the effective Majorana neutrino mass defined as

$$
\left|m_{\beta \beta}\right|=\left|\sum_{i=1,2,3} U_{e i}^{2} m_{i}\right| .
$$

This is also considered as the unique probe for the Majorana nature of neutrinos. There are many ongoing and upcoming experiments such as GERDA [25], CUORE [26], KamLand-Zen [27], GERDA Phase II [28], nEXO [29], which aim to achieve a sensitivity up to $0.01 \mathrm{eV}$ for $\left|m_{\beta \beta}\right|$.

In the NH case where $m_{1} \lesssim m_{2}<m_{3}$, it is useful to rewrite the heaviest states in terms of the lightest one and the mass squared differences as $m_{2}=\sqrt{m_{1}^{2}+\Delta m_{21}^{2}}$ and $m_{3}=\sqrt{m_{1}^{2}+\Delta m_{31}^{2}}$, where $m_{1}, m_{2}$ and $m_{3}$ are as given in eq. (3.9). By using the $3 \sigma$ allowed ranges of neutrino oscillation parameters from Ref. [16], we show in the left panel of Fig. 3 the correlation between the sum $\sum m_{i}$ and the three neutrino masses $m_{i=1,2,3}$ as a function of the lightest neutrino mass $m_{1}$. The predicted regions are as follows

$$
\begin{aligned}
& 0.00132 \lesssim m_{1}[\mathrm{eV}] \lesssim 0.03002,0.00840 \lesssim m_{2}[\mathrm{eV}] \lesssim 0.03130 \\
& 0.04948 \lesssim m_{3}[\mathrm{eV}] \lesssim 0.05871,0.05928 \lesssim \sum m_{i}[\mathrm{eV}] \lesssim 0.11966
\end{aligned}
$$




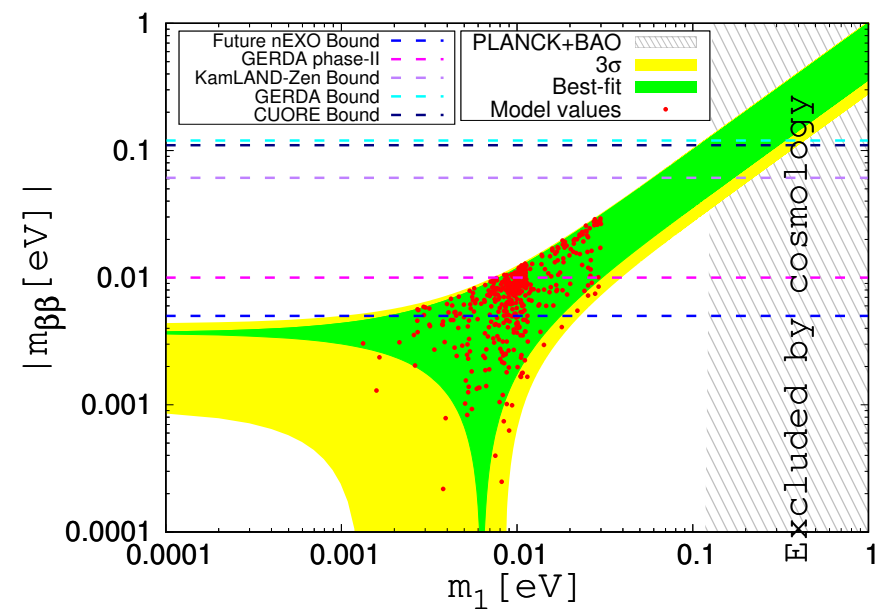

FIGURE 4: $\left|m_{\beta \beta}\right|$ as a function of $m_{1}$. The vertical filled area presents the upper limit on the sum of the three light neutrino masses from Planck+BAO data.

We find that the obtained range of $\sum m_{i}$ is below the upper bound given by the Planck collaboration with a lower bound given by $\sum m_{i} \gtrsim 0.05928 \mathrm{eV}$. Even though this lower limit is small, it can be tested by future experiments such as CORE+BAO aiming for a bound on $\sum m_{i}$ around $0.062 \mathrm{eV}$ [30]. In the right panel of Fig. 3, we show the allowed region of the lightest neutrino mass $m_{1}$ and the effective electron antineutrino mass $m_{\beta}$. In this plot, we first used the definition of $m_{\beta}$ given in eq. (4.18) and we replaced $U_{e i}$ by the elements of the first row of $\mathrm{TM}_{2}$ given in eq. (1.1). Then, by taking into consideration the $3 \sigma$ allowed ranges of neutrino oscillation parameters as well as the neutrino mass constraints in eq. (4.20), we obtain the following range for $m_{\beta}$

$$
0.00873 \lesssim m_{\beta}[\mathrm{eV}] \lesssim 0.03135
$$

The values in this range are too small compared to the KATRIN sensitivity $(\sim 0.2 \mathrm{eV})$ and the expected sensitivity from HOLMES experiment $(\sim 0.1 \mathrm{eV})$ [31], and thus, they can not be tested in the foreseeable future. On the other hand, as shown by the blue dashed line in the right panel of Fig. 3, the upper limit in 4.21 is close to the anticipated sensitivity of Project 8 collaboration (0.04 eV) [32] and hence, can be reached by future experiments targeting a more enhanced sensitivities.

By repeating the same exercise as before, we first use the definition of $\left|m_{\beta \beta}\right|$ given in eq. (4.19) and we replaced $U_{e i}$ by the elements of the first row of $\mathrm{TM}_{2}$ given in eq. (1.1). Then, we plot in Fig. 4 the the effective Majorana neutrino mass $\left|m_{\beta \beta}\right|$ as a function of the lightest neutrino mass $m_{1}$ where the Majorana phases $\alpha_{21}$ and $\alpha_{31}$ are allowed to vary in the range $[0 \rightarrow 2 \pi]$. The horizontal dashed lines in this figure denote the $\left|m_{\beta \beta}\right|$ sensitivity from several $0 v \beta \beta$ decay experiments. Our predicted values (red dots) for $\left|m_{\beta \beta}\right|$ that satisfy constraints from oscillation experiments are given by the following range

$$
0.00021 \lesssim\left|m_{\beta \beta}[\mathrm{eV}]\right| \lesssim 0.02923
$$

From this figure, we observe that all the allowed points are below the current sensitivities from GERDA, CUORE, and KamLandZen experiments, while most of these points are scattered within the future upper bounds of GERDA Phase II and nEXO experiments, and thus, our predicted values of $\left|m_{\beta \beta}\right|$ can be tested by these experiments.

\section{CONCLUSION}

In this work, we have constructed a neutrino flavor model based on the $\Delta(54)$ discrete group that accommodates the observed neutrino masses and mixing. We have shown that the combination of type II seesaw mechanism with $\Delta$ (54) flavor group in the same framework lead to a simple neutrino mass matrix with one texture zero which restricts the number of free parameters to three parameters. Moreover, the obtained neutrino mass matrix has the magic symmetry and thus, predicts a rich phenomenology provided by the trimaximal mixing of neutrinos. In particular, the most interesting results of our model is that it predicts the normal hierarchy for neutrino masses and the lower octant for the atmospheric angle. We have studied the phenomenological implications associated with neutrino masses where we found that the range of the effective Majorana neutrino mass $m_{\beta \beta}$ is within the reach of future experiments while the obtained range of the electron neutrino mass $m_{\beta}$ is far from current and future experimental bounds. Many issues remain to be studied in this promising model, such as the quark and the scalar sectors and their phenomenology. On the other hand, since we found that $C P$ is always violated in the current model, it would be useful to examine other neutrino related topics like the triplet contribution to the lepton asymmetry of the Universe; we leave detailed investigations of these subjects to future work. 


\section{References}

[1] T. Kajita, Rev. Mod. Phys. 88, 030501 (2016).

[2] A. B. McDonald, Rev. Mod. Phys. 88, 030502 (2016).

[3] P. Minkowski, Phys. Lett. 67B, 421 (1977); M. Gell-Mann, P. Ramond, and R. Slansky, Conf. Proc. C790927, 315 (1979).

[4] J. Schechter and J. W. F. Valle, Phys. Rev. D 22, 2227 (1980).

[5] R. Foot, H. Lew, X-G He and G. Joshi, Z. Phys. C 44 (1989) 441.

[6] E. Ma, Phys. Rev. D 73 (2006) 077301.

[7] S. Baumholzer, V. Brdar, P. Schwaller, and A. Segner, JHEP 09, 136 (2020); A. Ahriche, A. Jueid, S. Nasri, Phys. Rev. D 97 (2018) $095012 ;$ A. Ahriche, A. Arhrib, A. Jueid, S. Nasri, A. de la Puente, Phys. Rev. D 101 (2020) 035038.

[8] E. Ma, Phys. Lett. B 671 (2009) 366, E. Ma, A. Natale, A. Rashed, Int. J. Mod. Phys. A 27 (2012) 1250134; D. Meloni, S. Morisi, E. Peinado, Phys. Lett. B 703 (2011) 281-287; M. Loualidi and M. Miskaoui, Nucl. Phys. B961, 115219 (2020).

[9] N. Haba, A. Watanabe and K. Yoshioka, Phys. Rev. Lett. 97 (2006) 041601; X. G. He and A. Zee, Phys. Lett. B 645, (2007) 427; W. Grimus and L. Lavoura, J. High Energy Phys. 0809, 106 (2008); H. Ishimori, Y. Shimizu, M. Tanimoto and A. Watanabe, Phys. Rev. D 83, 033004 (2011); Y. Shimizu, M. Tanimoto, A. Watanabe, Prog. Theor. Phys. 126 (2011) 81-90; X. G. He, A. Zee, Phys. Rev. D 84, 053004 (2011); I. de M. Varzielas and D. Pidt, J. High Energy Phys. 03, 065 (2013).

[10] P. F. Harrison, D. H. Perkins, and W. G. Scott, Phys. Lett. B 530, 167 (2002).

[11] P. H. Frampton, S. L. Glashow, and D. Marfatia, Phys. Lett. B 536, 79 (2002).

[12] Z. -z. Xing, Phys. Lett. B 530, 159 (2002).

[13] E. Lashin and N. Chamoun, Phys. Rev. D 85, 113011 (2012).

[14] R. R. Gautam, Phys. Rev. D 97, 055022 (2018).

[15] C. S. Lam, Phys. Rev. D 74, 113004 (2006)

[16] I. Esteban, M. C. Gonzalez-Garcia, M. Maltoni, T. Schwetz, and A Zhou, J. High Energy Phys. 09 (2020) 178.

[17] H. Ishimori, T. Kobayashi, H. Ohki, H. Okada, Y. Shimizu, and M. Tanimoto, Prog. Theor. Phys. Suppl. 183, 1 (2010).

[18] M. S. Boucenna, S. Morisi, E. Peinado, J. W. F. Valle and Y. Shimizu, Phys. Rev. D 86, 073008 (2012).

[19] C. D. Froggatt and H. B. Nielsen, Nucl. Phys. B147, 277 (1979).

[20] R. Ahl Laamara, M. A. Loualidi, and E. H. Saidi, Phys. Rev. D 93, 113005 (2016).

[21] M. A. Ouahid, M. A. Loualidi, R. Ahl Laamara, E. H. Saidi, Phys. Rev. D 102, 115023 (2020).

[22] M. Tanabashi et al. (Particle Data Group), Phys. Rev. D 98, 030001 (2018).

[23] Planck Collaboration and N. Aghanim et al., arXiv:1807.06209 (2018).

[24] S. Mertens, Phys. Procedia 61, 267 (2015).

[25] M. Agostini et al. (GERDA Collaboration), Phys. Rev. Lett. 111, 122503 (2013).

[26] C. Alduino et al. (CUORE Collaboration), Phys. Rev. Lett. 120, 132501 (2018).

[27] A. Gando et al. (KamLAND-Zen Collaboration), Phys. Rev. Lett. 117, 082503 (2016).

[28] M. Agostini et al. (GERDA Collaboration), Nature 544 (2017) 47-52.

[29] J. B. Albert et al. (nEXO Collaboration), Phys. Rev. C 97, 065503 (2018).

[30] CORE Collaboration, E. Di Valentino et al., J. Cosmol. Astropart. Phys. 1804, 017 (2018).

[31] HOLMES Collaboration, B. Alpert et al., Eur. Phys. J. C 75, 112 (2015).

[32] Project 8 Collaboration, A.A. Esfahani, et al., J. Phys. G 44 (2017) 054004. 